\title{
HDOT - an Approach Towards Productive Programming of Hybrid Applications
}

\author{
Jan Ciesko ${ }^{\mathrm{a}}$, Pedro J. Martínez-Ferrer ${ }^{\mathrm{a}, *}$, Raúl Peñacoba Veigas ${ }^{\mathrm{a}}$, Xavier \\ Teruel $^{\mathrm{a}}$, Vicenç Beltran ${ }^{\mathrm{a}}$ \\ ${ }^{a}$ Barcelona Supercomputing Center (BSC)
}

\begin{abstract}
A wealth of important scientific and engineering applications are configured for use on high performance computing architectures using functionality found in the MPI specification. This specification provides application developers with a straightforward means for implementing their ideas for execution on distributed-memory parallel processing computers. OpenMP directives provide a means for operating on shared-memory regions of those computers. With the advent of machines composed of many-core processors, the strict synchronisation required by the bulk synchronous parallel (BSP) communication model can hinder performance increases. This is due to the complexity to handle load imbalances, to reduce serialisation imposed by blocking communication patterns, to overlap communication with computation and, finally, to deal with increasing memory overheads. The MPI specification provides advanced features such as non-blocking calls or shared memory to mitigate some of these factors. However, applying these features efficiently usually requires significant changes on the application structure.

Task parallel programming models are being developed as a means of mitigating the abovementioned issues but without requiring extensive changes on the application code. In this work, we present a methodology to develop hybrid applications based on tasks called hierarchical domain over-decomposition with tasking (HDOT). This methodology overcomes most of the issues found on MPIonly and traditional hybrid MPI+OpenMP applications. However, by emphasising the reuse of data partition schemes from process-level and applying them to task-level, it enables a natural coexistence between MPI and shared-memory programming models. The proposed methodology shows promising results in terms of programmability and performance measured on a set of applications.
\end{abstract}

Keywords: concurrency, parallel programming, hybrid programming, MPI,

(C) 2019. This manuscript version is made available under the CC-BY-NC-ND 4.0 license https://creativecommons.org/licenses/by-nc-nd/4.0/

«m Published journal article available at https://doi.org/10.1016/j.jpdc.2019.11.003

* Corresponding author

Email address: pedro.martinez-ferrer@bsc.es (Pedro J. Martínez-Ferrer) 
OpenMP, OmpSs-2

\section{Introduction}

Non-coherent, distributed memory is a common characteristic of modern HPC systems. Such memory organisation allows to assemble large systems from commodity components which reduces cost, increases versatility of use and offers flexibility to quickly adapt to application trends. This is referred to as cluster architecture.

MPI 1 is a commonly used programming specification of such systems. In this scenario, programmers work at the process level (i.e. MPI rank), where each of these processes has its memory address space and therefore require explicit communication for data exchange. The programmer uses functions such as MPI_SEND or MPI_RECV to exchange and synchronise data between processes. In other words, the developer implements data distribution and coherence, such that this functionality becomes part of the algorithm. Designing algorithms with concurrency in mind makes software development more difficult, which can be overwhelming for novice programmers. However, it turns out that the nature of MPI, and imperative parallel programming models in general, obliges the developer to consider concurrency early on in the development. Typically this results in good design that favors concurrency and scalability. As a consequence, many MPI-only applications, i.e. those whose parallelism is implemented using functionality from the MPI specification, tend to achieve better performance scalability compared to applications using incremental parallelism.

Algorithmic design for performance and scalability typically follows one rule: "always keep the processors busy advancing the computation". Under the hood this means: (i) implementing ordering of computation and communication for overlaps, (ii) ensuring balanced execution, (iii) keeping overheads low and, finally, (iv) creating enough parallelism. It turns out that this is becoming a real challenge since the increasing number of processor cores per nod ${ }^{1}$ makes it more and more difficult to achieve scalability on these systems. This is due to the fact that, as the number of processes within the same node increases, efficiency of each MPI process drops [2. The main causes for this behaviour are data overheads, system heterogeneity, load imbalances and suboptimal communication patterns, all of which are difficult to eliminate or optimise. In such cases, it can be beneficial to combine the message-passing programming model to exploit internode parallelism with another shared-memory programming model to exploit intranode parallelism [3, 4.

\footnotetext{
${ }^{1}$ Currently, the Intel Skylake processor architecture supports up to 28 physical cores (the recently announced Cascade Lake processor will double this number) whilst the already available AMD EPYC 7742 CPU features 64 physical cores.
} 


\subsection{Are shared-memory programming models the right solution?}

On shared memory programming models, threads are building blocks to exploit parallelism within one MPI process. On a shared data environment, load balancing techniques can be efficiently implemented, there is no need to replicate extra data and all the threads have direct access to all the data environment of their MPI process. However, to benefit from threading, an existing MPIonly code must be adapted. A variety of ways exist to express concurrency on thread-level of which OpenMP is a particularly popular one (see Section 2.1). An application that combines multiple programming models is called hybrid application.

In practice, hybrid codes can indeed offer an improvement over MPI-only codes on modern many-core systems [5]. However, it is up to the skill of the programmer to combine both efficiently to achieve performance gains. In case of OpenMP, the programmer is in charge of creating, using and closing parallel regions (fork-join parallelism) and of synchronising threads with MPI calls to implement a correct communication pattern. The process of adding thread-level parallelism to an MPI application is characterised by a sequence of "looking for code sections with significant duration" and then "adding annotations to parallelise these code sections". This is strenuous and as a result, hybrid applications often end up with interleaved execution of concurrent computation phases (OpenMP) and communication phases with less parallelism (MPI), which limits the maximum speed-up of the entire application in accordance to Amdahl's law. We call this two-phase programming.

It can be readily seen that it becomes necessary to define a methodology that helps developers to avoid the limitations of two-phase programming, allowing a more natural coexistence between MPI and shared-memory programming models.

\subsection{From processes to tasks}

In this work we present HDOT, hierarchical domain over-decomposition with tasking, a methodology that simplifies hybrid programming and improves the execution performance of such applications.

HDOT applies the domain decomposition of an existing MPI application at process-level to thread-level. At this thread-level, domains are decomposed into subdomains and executed by tasks. HDOT maximises pattern and code reuse and adds the advantages of tasking. Tasking simplifies the development of hybrid applications by eliminating two-phase programming and by reducing the complexity of synchronisation of parallel work and MPI calls.

The HDOT methodology defines a top-down approach where the developer uses tasks to encapsulate work from a coarse-grained level down to tasks as small as individual send and receive MPI messages. This includes the definition of subdomains and support of common algorithmic patterns such as reductions and global variables.

The OmpSs-2 programming model (see Section 2.2 eliminates the notion of threads and emphasises the use of a task as a building primitive for concurrency. Tasks are expressed declaratively and can encapsulate computation 
and MPI communication alike. The task-aware MPI (TAMPI) library (see Section 2.3 ensures the correct execution of MPI calls within tasks. Finally, the programming model's support for data-flow programming allows a streamlined execution of computation and communication tasks.

In the next chapter we provide useful background information on today's challenges of MPI and hybrid programming, give a short introduction to OmpSs2 and TAMPI, and discuss the related work. Next, we present the aforementioned methodology and apply it on a set of applications. Finally, we present performance results achieved with MPI+OmpSs-2, MPI+OpenMP and MPIonly implementations.

\section{Background}

\subsection{MPI and OpenMP}

Threading allows to reduce the number of MPI processes per node while maintaining the same degree of concurrency of the application. OpenMP [6] is a widely known multi-threaded shared-memory programming model. It allows to parallelise applications by using a set of compiler directives, library routines and environment variables. OpenMP is flexible and supports most parallel patterns: from the traditional work-sharing approach to the OpenMP accelerator and tasking models. However, taking a closer look at existing codes shows that the prevalent use of OpenMP is the expression of data-parallel algorithms with an execution model called fork-join. Since OpenMP 3.0 [7, it is possible to express dynamic task parallelism through task generating constructs (including task, taskgroup, taskyield and taskwait). A task is a unit of work used to express portions of code that could be executed concurrently by the participant threads in accordance to certain restrictions.

Code 1: Representative MPI-only application with interleaved point-to-point and collective communication.

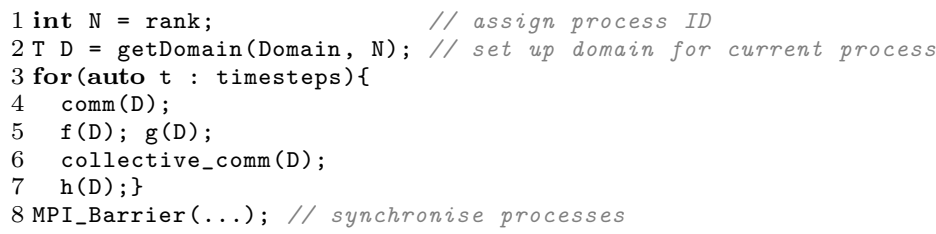

Code 1 shows an MPI-only application where a sequence of functions is called in a simulation loop over a predefined set of time steps. In this code example, $\mathrm{D}$ is an object that handles data associated with the current MPI domain whilst comm and collective_comm contain point-to-point and collective MPI communication, respectively. Once the execution of the simulation loop finishes, processes synchronise at an MPI_Barrier. Code 2 shows the same algorithm enhanced with OpenMP. As the number of processes is reduced in 
(a)

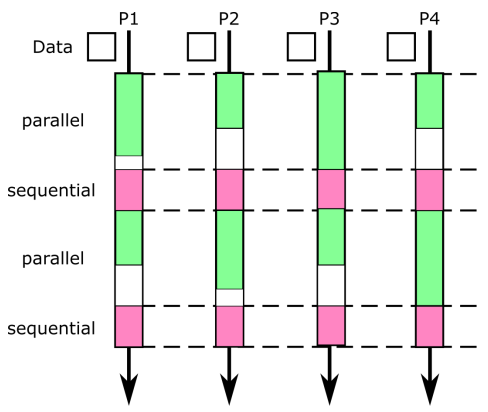

(b)

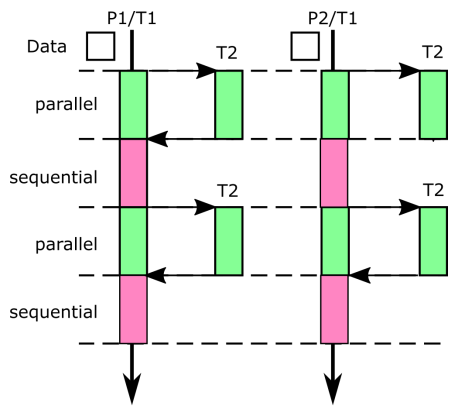

Figure 1: Execution diagram of an application with interleaved communication and computation using (a) MPI-only and (b) MPI+OpenMP implementations. The hybrid version illustrates the fork-join parallel execution of the compute functions (parallel) and its ability to balance load among threads.

favour of threading, the compute functions $f, g$ and $h$ are now placed inside a parallel region and executed on each thread (fork). Threaded execution requires to adjust the implementations of these functions, hence we name them f_parallel, g_parallel and h_parallel. At the end of each parallel region, an implicit barrier synchronises threads (join). Such thread synchronisation is necessary in order to maintain the correct ordering of communication and computation.

Code 2: A hybrid application implemented with MPI and OpenMP showing two parallel regions and the respective synchronisation points at thread- and process-level.

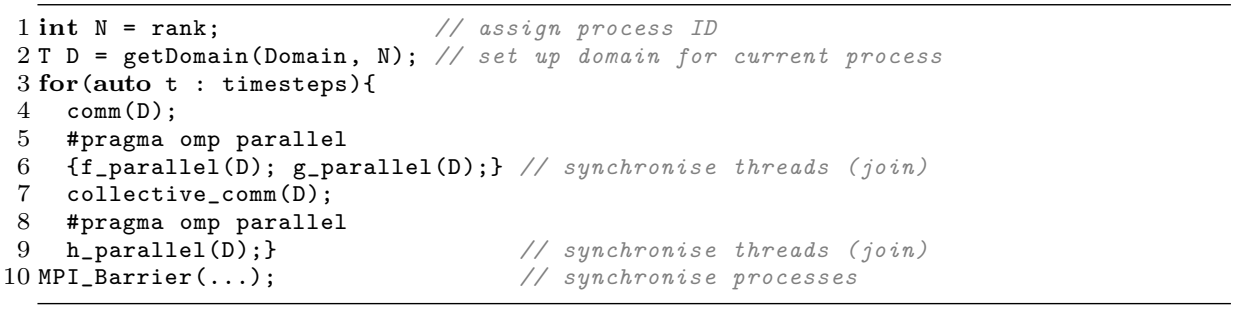

Figure 1 shows an execution diagram of the fork-join parallelism of MPI-only and MPI+OpenMP implementations. The execution diagram also illustrates, on the one hand, the additional amount of data copies of the MPI-only version (multiple data objects) and, on the other hand, the ability of load-balancing of the hybrid version.

\subsection{Tasking with OmpSs-2}

OmpSs-2 8] (OMP SuperScalar v2) is a high-level, task-based, parallel programming model for shared-memory systems developed at the Barcelona Su- 
percomputing Center (BSC). It consists of a language specification, a source-tosource compiler for $\mathrm{C}, \mathrm{C}++$ and Fortran as well as a runtime. OmpSs- 2 defines a set of directives for a descriptive expression of tasks. Further, it allows the programmer to annotate task parameters with in, out and inout clauses that correspond to the input, output or the combination of input-output access type semantic of these parameters within a task. Each access type clause receives a list of parameters:

\#pragma oss task [in(par-list) | out(par-list) | inout(par-list)]

The access type establishes a producer-consumer relationship between tasks, also called task dependency or data-flow. With this information the runtime is capable of scheduling tasks automatically that maintain correctness of code while alleviating the programmer of implementing manual synchronisation. Furthermore, the taskwait construct allows task synchronisation and instructs the calling thread to wait on all previously created tasks. While this is similar to tasking in the recent specification of OpenMP, the OmpSs-2 runtime implements a different execution model.

In OmpSs-2, every application starts with a predefined set of execution resources, that is, an explicit parallel region does not exist. This view avoids the exposure of threading to the programmer as well as the requirement to handle an additional scope as in OpenMP. At compile time, the OmpSs-2 compiler processes pragma annotations and generates an intermediate code file. This file includes both user code and additional code for task generation, synchronisation and error handling. In the final step of compilation, OmpSs-2 invokes the native compiler to create a binary file.

At runtime, the main function is executed, which creates tasks and stops at (explicit or implicit) synchronisation points. Task creation is composed of two parts: (i) the creation of the task object itself that carries all its descriptive information and (ii) its data dependencies. Once a task object has been created, the runtime inspects the dependency graph to determine the relationship with respect to previously created tasks. If a dependency has been found, a representative node is added to the graph. In the opposite case, the task is placed into a ready-queue. Tasks in the ready-queue are picked up by worker threads, removed from the queue and executed.

Interestingly, one question arises: is it possible to leverage the dynamic, dataflow driven task execution in OmpSs-2 to improve the performance of MPI-only applications? In the following section we present TAMPI, a task-aware MPI library, which enables a natural coexistence of the OmpSs-2 shared-memory programming model with the MPI distributed-memory specification.

\subsection{Task-aware MPI}

The task-aware MPI (TAMPI) library [9, 10] extends the functionality of standard MPI libraries by providing new mechanisms for improving the interoperability between parallel task-based programming models, such as OpenMP or OmpSs-2, and both blocking and non-blocking MPI operations. 
By following the MPI specification, programmers must pay close attention to avoid deadlocks that may occur in hybrid applications (e.g. MPI+OpenMP) where MPI calls take place inside tasks. This is given by the out-of-order execution of tasks that consequently alter the execution order of the enclosed MPI calls. The TAMPI library ensures a deadlock-free execution of such hybrid applications by implementing a cooperation mechanism between the MPI library and the parallel task-based runtime system.

TAMPI provides blocking [9] and non-blocking [10] modes. The blocking mode targets the efficient and safe execution of blocking MPI operations (e.g. MPI_Recv) from inside tasks, while the non-blocking mode focuses on the efficient execution of non-blocking or immediate MPI operations (e.g. MPI_Irecv), also from inside tasks.

TAMPI is compatible with mainstream MPI implementations that support the MPI_THREAD_MULTIPLE threading level, which is the minimum requirement to provide its task-aware features. This library is a refinement of the hybrid MPI+SMPSs approach presented in [11. We consider it as a key feature towards achieving performance and scalability of hybrid applications on modern systems today. It is worth mentioning that it is still the user's responsibility to prevent races when threads within the same application post conflicting communication calls. Furthermore, we would like to emphasise that the development of hybrid applications based on tasks can be fully achieved without using this library, but at the expense of a greater programming effort. This is briefly discussed in Section 4.3

\subsection{Related work}

MPI-based parallelisation approaches try to improve performance and scalability of applications by overlapping communication and computation [12, 13,14 and by accelerating the execution of the critical path of the program [15, 16, 17, 18.

Although the MPI-only approach is still one of the most popular among the set of parallelisation alternatives within the high performance computing community, programmers will have to exploit hybrid solutions (MPI+X) that allow a better use of hardware resources in order to reach the so-called exascale era [19. In general, these hybrid approaches also try to enhance the application behaviour by overlap and critical path programming techniques [5, 11.

SMPSs [1] enables communication and computation overlap by taskifying MPI calls and adding a restart functionality which allows re-scheduling tasks waiting for a certain condition (similarly to a mandatory taskyield with extra scheduler semantics). In our approach, this functionality is transparently provided by the TAMPI library. Furthermore, in SMPSs the programmer needs to explicitly split a blocking call into a non-blocking call to issue the communication request and wait for the data, while TAMPI does not require this transformation from the programmer.

HCMPI [5] unifies the Habanero-C intranode task parallelism with MPI internode parallelism and extends the tasking model by allowing regular computation tasks to create asynchronous communication tasks. In HCMPI's data-flow 
model, synchronisation between computation and communication tasks can be achieved through the specific await clause and other specific MPI-like services (e.g. wait, waitall or waitany).

Both SMPSs and HCMPI require a communication thread dedicated to execute MPI calls, while our methodology does not force how to implement the communication progress. Moreover, both approaches are focused exclusively on the communication pattern, independently of how the computational decomposition is carried out. We consider that these two elements (computation and communication) are so dependent the one to the other that they should be interrelated when applying any parallel decomposition approach.

The methodology presented in this work is also aligned with those build on top of task-based runtime systems (also referred to as user-level threads) as a second level of parallelism. The main reason for using this approach is that it indeed represents a more natural way to convert MPI code to tasking of a shared-memory parallel programming model. Among those related works proposing task-parallelism, we would like to highlight the task parallel overdecomposition (TPOD) approach [4] due its similarity to the present work.

TPOD combines computation and communication operations in the same task in a way that maintains the traditional MPI coding style. This strategy is based on over-decomposing the domain that has been assigned to a set of cores (i.e. MPI processes) into a set of smaller subdomains (i.e. tasks). The main difference of TPOD tasks with respect to regular tasks is found when the code reaches a blocking communication call. In this situation the task may be swapped out, allowing another task to be swapped in and continue with the execution. At some point, the communication-blocked task will be swapped back in and continue with its normal execution.

Although the TPOD approach also uses tasks to overlap computation and communication phases, it relies on global barriers between different iterations. This strategy restricts the degree of potential concurrency that the application can reach, being better to rely on fine-grained synchronisation such as the task dependency system. MPI+OmpSs-2 naturally exploits nesting capabilities to balance the trade-off between grain size and the degree of concurrency based on a top-down approach.

\section{HDOT: Hierarchical Domain Over-decomposition with Tasking}

HDOT leverages the parallelisation and domain decomposition schemes of the original MPI application by promoting their reuse on task-level. In this scheme, domains are split hierarchically, first at process-level (MPI), down to task-level (shared memory) at which domains are referred to as subdomains.

Applying a hierarchical over-decomposition with tasking follows a set of underlying ideas. Firstly, it minimises requirements for code changes and allows reuse of original MPI code and its data partitioning. Secondly, it decouples tight synchronisation between units of work and MPI communication. And lastly, it emphasises the use of a top-down approach where concurrency is added on differ- 
ent nesting levels to create opportunity for concurrency at a small development effort. We discuss this in three steps as follows.

\subsection{Taskifying code}

Let us continue with Code 2 In this code example, the maximal speedup is limited by the fork-join pattern with sequential sections and the tight synchronisation between computation and communication. To apply the HDOT methodology, we start with the original, MPI-only Code 1. In the first step, we taskify code sections. That is, we start adding the OmpSs-2 task pragmas to the code, stating that the enclosed code is adept for concurrent execution. It is important to point out that, by taskifying most of the application code, the number of synchronisation points gets reduced. The execution of tasks in the correct order is guaranteed by following the application data-flow. Code 3 is based on the previous example but now includes tasks with their respective data dependencies.

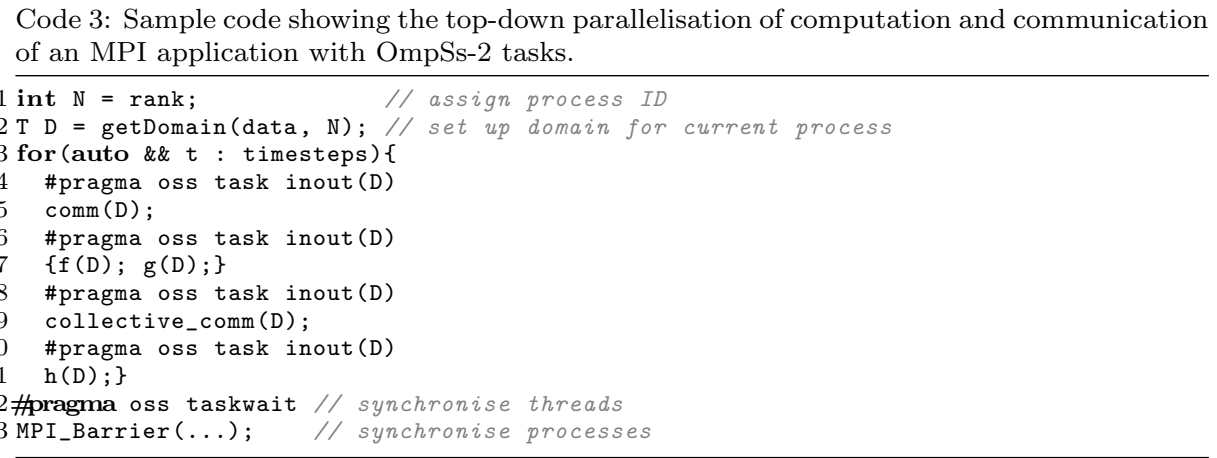

In this case, the non-blocking execution of MPI calls by including them in tasks and in the application data-flow is an important feature towards scalability and programmability. In Code 3 , dependencies of the type inout serialise the execution of the three tasks. The code thus requires a finer task granularity via subdomains, which is discussed in the following section.

\subsection{Adding subdomains}

To increase the degree of concurrency of the application shown in Code 3 we implement what we refer to as a domain over-decomposition that splits domains into subdomains of arbitrary sizes. On the one hand, a domain is a physical data partition implemented originally by an MPI application; on the other hand, a subdomain is a virtual data partition implemented for the use of a shared-memory programming model with the aim of increasing the nodelevel parallelism. Subdomains follow the same idea of data partitioning found on process level. In case of subdomains, tasks and task functions operate on 
smaller, process-local data with occasional communication. The implementation of those functions remains unchanged.

Code 4: Subdomains allow to reuse the domain partitioning at process-level but also require to check for boundary subdomains and the use of weak dependencies.

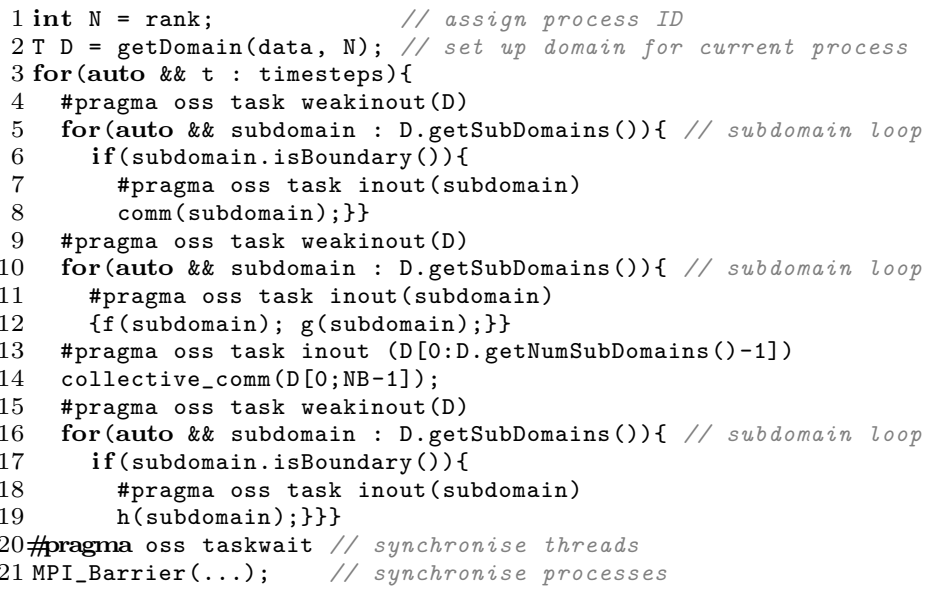

Code 4 shows the code changes made in order to accommodate subdomains. It can be seen that we have added an additional task nesting level, i.e. subdomain loops. On the inner nesting level, new tasks are created in for-loops where the number of inner tasks corresponds to the number of subdomains. To add these tasks to the data-flow of the application, each newly created task defines an inout dependency over an individual subdomain. However, since encapsulating tasks from the original code would still serialise the execution between loops, we apply the concept of weak dependencies via the prefix weak.

Weak dependencies [20] is a key feature of the OmpSs-2 programming model to allow a top-down parallelisation of code. They break coarse-grained dependencies between tasks under the assumption that inner tasks will fulfill the dependency requirements. This results in fine-grained dependencies between tasks where a communication task over a subdomain can be immediately executed once the prior computation task over that subdomain has finished. Towards the end of the code sample, a collective MPI operation accesses all subdomains and therefore defines an inout dependency over all of them. Furthermore, since not all subdomains are equal in their correspondence to the geometric position in the original domain, we have added the condition isBoundary to check whether the subdomain type requires to communicate halo data with MPI.

Finally, it is worth noting two important aspects about subdomains and their associated task granularity. Firstly, on task-based runtime systems the number of tasks (which is inversely proportional to the task granularity) should be preferably larger than the number of cores (even an order of magnitude 
larger) available per MPI process to guarantee good scalability properties, that is, to guarantee enough parallelism without incurring into runtime scheduling costs. On the other hand, network saturation could become an issue with a fine granularity, for instance, when tasks communicate message sizes smaller than $1 \mathrm{~KB}$, approximately, on an InfiniBand network. In such case, the task granularity associated with communication must be increased whilst maintaining the same granularity for computation tasks.

\subsection{Including support for common programming patterns}

In many scientific codes, developers use reductions and global (static) variables. Reductions are operations that possess an identity element and can be processed in parallel since each concurrent unit of work can initialise a private set of operands to the neutral element following the same approach adopted by OpenMP. We make use of these properties in HDOT and show how reductions are computed on task- and process-level. In those cases the reduction value represents either an intermediate result at task-level or a final result on process-level.

Code 5: Support for reductions and static variables requires the addition of the reduction clause and local variables, respectively.

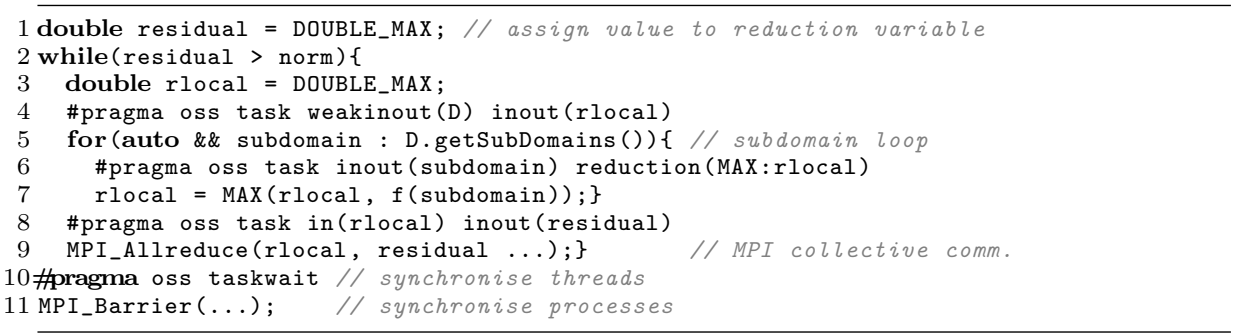

Code 5 is a continuation of the code samples previously shown; however, we have removed irrelevant code lines. The reduction clause instructs the runtime system to provide a thread-safe storage such that the execution of any two concurrent tasks is data-race free. Once all participating tasks complete, the task calling the MPI_Allreduce function can be executed. In OmpSs-2, a reduction creates an input-output dependency implicitly.

In order to maintain the dependency between computation and communication tasks, we have added a stack-local reduction variable called rlocal. This creates a dependency between the computation tasks that perform the reduction and the communication task that perform the MPI_Allreduce which defines an input over that variable.

Algorithms often use global variables to store the state of the simulation or geometric properties describing a domain. However, an over-decomposition of the simulation domain requires stack-local variables that hold pointers to such data structure for each task. For this purpose, Codes 45 implement the 
functions getSubDomains and getNumSubDomains that return a set of subdomains and their number, respectively. Using pragma annotations similar to firstprivate for custom data types is not optimal as this typically invokes the copy constructor of that object resulting in replication of potentially large data.

\section{Applications}

In this section we present the performance results obtained for two benchmarks, Heat2D and HPCCG, and one large application, CREAMS. The results were obtained on the MareNostrum 4 supercomputer located at the Barcelona Supercomputing Center (BSC). Each node of MareNostrum 4 is equipped with a dual-socket Intel Platinum $8160 \mathrm{CPU}$ featuring 24 cores per processor, $96 \mathrm{~GB}$ of main memory and Intel's Omni-Path HFI interconnect network.

\subsection{Heat2D benchmark}

Heat2D is a popular benchmark that simulates heat diffusion in two dimensions. At each time step, a blocked Gauss-Seidel iterative solver approximates a solution of the Poisson equation. The continuous problem is discretised with finite differences. The resulting calculation for each discrete point is defined as: $U_{i, j}=\left(U_{i+1, j}+U_{i-1, j}+U_{i, j+1}+U_{i, j-1}\right) / 4$.

Heat2D is characterised by its stencil operation where each point update requires the access to the corresponding left, right, top and bottom neighbouring points.

Code 6: Pseudocode of the Heat2D benchmark.

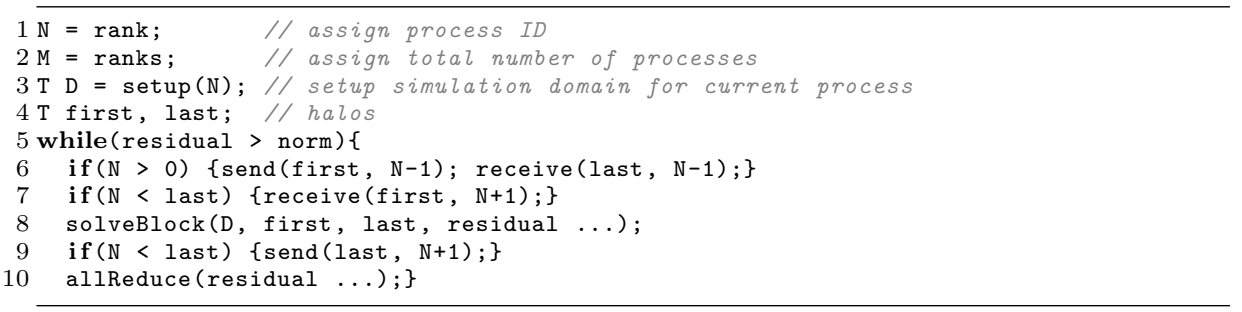

Code 6 gives a basic idea of how the heat benchmark is implemented, including the communication of halos and the actual computation carried out by the function solveBlock, whilst Figure 2a shows the stencil operation and a visual representation of the progression of the algorithm mapped over the same data set used for benchmarking later in this section. In this figure, the simulation space is divided in 4 MPI domains (ranks). During execution, each MPI rank contains four subdomains (blocks) and each subdomain is updated by one OmpSs-2 task. Dependencies that originate from the stencil operation for each element can be equally applied to define dependencies at block or process 
(b)

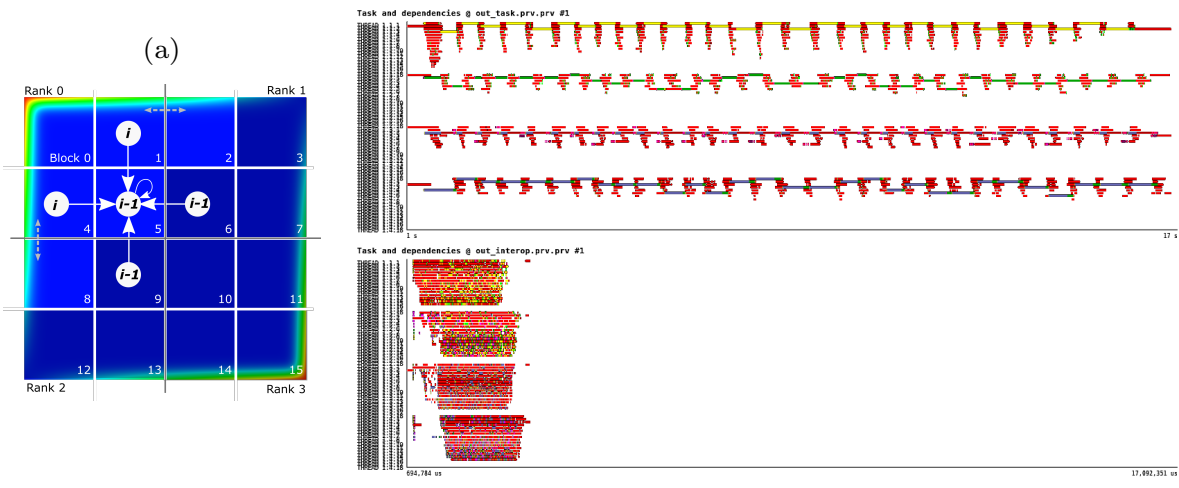

Figure 2: (a) Heat2D stencil operation results in a wave-front progression on process- and thread-level (its efficient execution requires fine-grained dependency resolution as well as computation and communication overlapping); (b) Heat2D traces on four nodes of MareNostrum 4 show massive differences in resource utilisation and performance between a traditional forkjoin approach using MPI+OpenMP (top) and our HDOT implementation using MPI+OmpSs2 (bottom).

\begin{tabular}{cccrccc}
\hline Nodes & Perf.M+OSs2 & Perf.M+OMP & Perf.M & S.M+OSs2 & S.M+OMP & S.M \\
\hline 1 & 6561 & 2836 & 2479 & 2.6 & 1.1 & 1.0 \\
2 & 12729 & 2096 & 4306 & 5.1 & 0.8 & 1.7 \\
4 & 25231 & 2134 & 6809 & 10.2 & 0.9 & 2.7 \\
8 & 48302 & 2757 & 9555 & 19.5 & 1.1 & 3.9 \\
16 & 99032 & 4259 & 11956 & 39.9 & 1.7 & 4.8 \\
32 & 145547 & 5839 & 13211 & 58.7 & 2.4 & 5.3 \\
\hline
\end{tabular}

Table 1: Heat2D execution performance measured in giga updates per second (more is better) for MPI+OmpSs-2 (M+OSs2), MPI+OpenMP (M+OMP) and MPI-only (M) implementations. Speed-up numbers (S) are normalised by the execution performance of the MPI-only version on one node. For the MPI-only version the number of MPI processes or ranks equals the total number of cores.

level. That is, MPI processes 1 and 2 can only start when blocks 1 and 4 of process 0 have finished computation and when the associated communication tasks (marked by the dotted double-sided arrows in Figure 2a) have completed as well. Hybrid code implementations of this particular benchmark (not shown here for the sake of conciseness) can be found in an early form in 9 .

Figure $2 \mathrm{~b}$ shows a trace of the execution of the Heat2D application on four nodes of the MareNostrum 4 supercomputer implemented with MPI+OpenMP (top) and MPI+OmpSs-2 (bottom). It can be readily seen that the implementation using OpenMP (fork-join execution model) is not capable of generating enough parallelism in order to maintain all the processor cores busy.

Table 1 shows the performance results for three parallel implementations of this application using up to 32 nodes. The implementation with OmpSs-2 and the TAMPI library allows to streamline the interleaved execution of computation 
and communication tasks shown in Code 6 and avoid any form of thread-local (OpenMP) or process-wide (MPI) synchronisation. On the other hand, the hybrid MPI+OpenMP implementation requires the presence of a thread barrier (\#pragma omp barrier) at the end of each loop that processes the local blocks (solveBlock). This way, any communication task for a particular halo can only be executed once computations of all blocks finish their execution. Similarly, the MPI-only implementation shows significant slow-downs due to the use of blocking MPI calls in order to maintain a correct ordering of computation and halo exchange. Authors' previous publications [9, 10] present a deeper study about the performance of each of these versions, including the results obtained by directly using the non-blocking MPI services.

\section{2. $H P C C G$ benchmark}

The high performance computing conjugate gradient (HPCCG) 21] benchmark consists of a synthetic sparse linear system that is mathematically similar to finite element, finite volume or finite difference discretisations of a threedimensional heat diffusion problem on a semi-regular grid. The problem is solved using domain decomposition with an additive Schwarz preconditioned conjugate gradient method where each subdomain is preconditioned using a symmetric Gauss-Seidel sweep.

The HPCCG benchmark generates a three-dimensional partial differential equation model problem and computes the preconditioned conjugate gradient iterations from the resulting sparse linear system. The global domain dimensions are $n_{x} \times n_{y} \times\left(n_{z} \times n_{p}\right)$, where $n_{x} \times n_{y} \times n_{z}$ are the local subgrid dimensions assigned to each MPI process and $n_{p}$ the number of MPI processes. In this work, each MPI process or domain is stacked in the $z$-direction.

Code 7: HPCCG body loop using an MPI-only implementation.

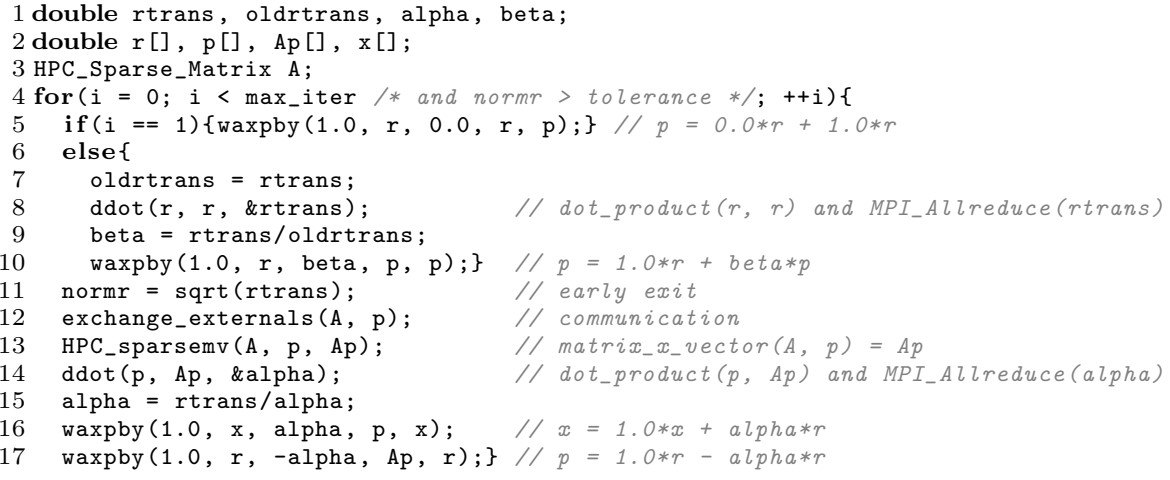

Halos are computed at the beginning of the program and exchanged at each iteration of the algorithm, as shown in line 14 of Code 7 . On the one hand, 


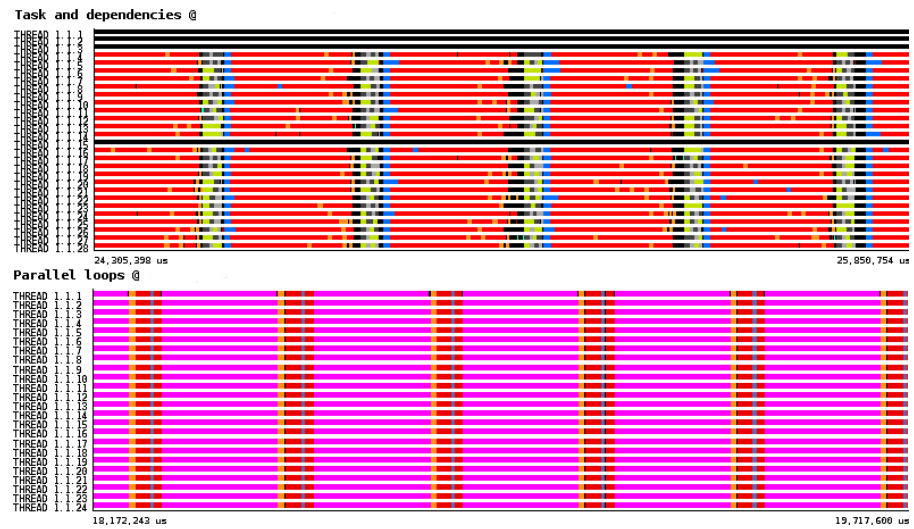

Figure 3: HPCCG paraver traces for MPI+OpenMP (top) and MPI+OmpSs-2 (bottom) implementations obtained with one node of MareNostrum 4.

the MPI+OpenMP approach consists of parallelising each operation using the pragma omp parallel for clause. On the other hand, the MPI+OmpSs-2 approach divides all the data structures in subdomains that can be executed in parallel via tasks.

Code 8: HPCCG (simplified) body loop using an MPI+OmpSs-2 implementation.

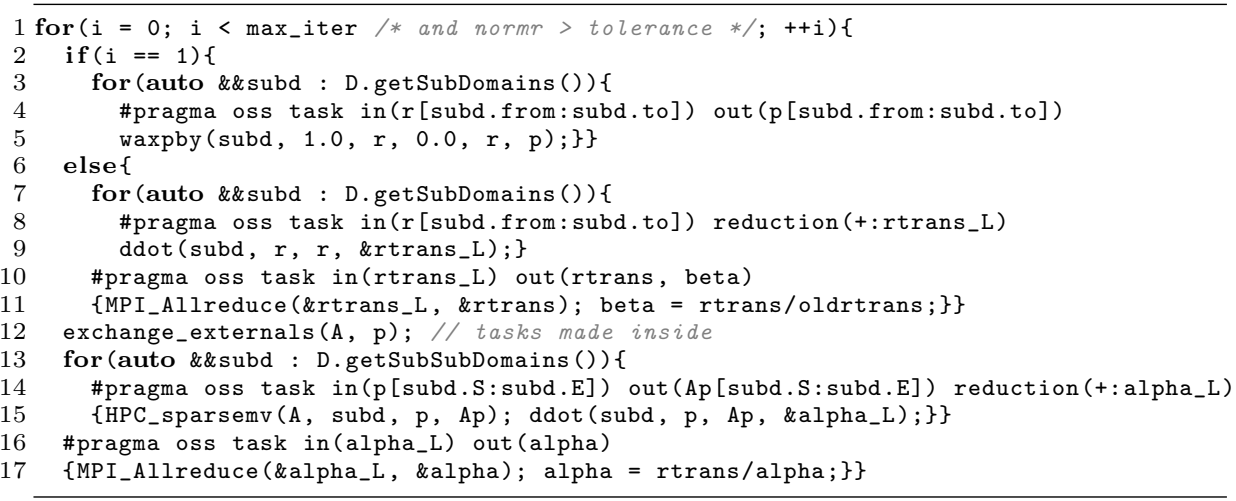

A snipet of the hybrid implementation of HPCCG using HDOT is illustrated in Code 8. It is worth noting that some variables, such as rtrans and alpha, need to be reduced in order to share them with other MPI processes. Moreover, it is necessary to take out the call to MPI_Allreduce inside the function ddot due to the need of a local reduction. The most computationally demanding operation, i.e. HPC_sparsemv, has been further divided by using nesting within subdomains, allowing to expose more parallelism.

Figure 3 shows a trace of HPCCG implemented with MPI+OpenMP fol- 
(a)

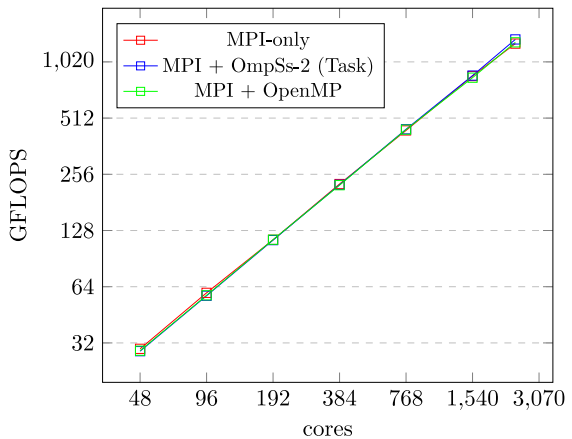

(b)

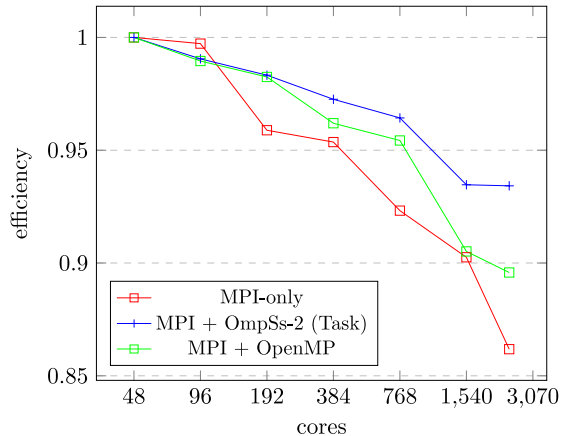

Figure 4: (a) HPCCG performace and (b) efficiency for different parallel implementations using up to 50 nodes (2400 cores) of MareNostrum 4.

lowing a two-phase approach (top) and MPI+OmpSs-2 following the HDOT approach (bottom). The MPI+OmpSs-2 version has a total of 32 subdomains, where nesting is applied on the HPC_sparsemv operation in order to feed the 48 CPUs available in one node.

Figures $4 \mathrm{a} 4 \mathrm{~b}$ show the performance and efficiency of HPCCG for three parallel implementations running on up to 50 nodes of MareNostrum 4. The MPI+OmpSs-2 version allows interleaving computation between subdomains by using the TAMPI library thus avoiding unnecessary waits, whilst the OpenMP version must wait for each parallel computation due to an implicit fork-join barrier.

\subsection{CREAMS application}

The compressible reactive multi-species (CREAMS) partial differential equation solver [22] is a computational fluid dynamics application that solves the full Navier-Stokes equations associated to multi-especies gas mixtures. CREAMS is an MPI-only application written in Fortran and based on the finite difference method) that is employed for direct numerical simulations and large eddy simulations. This application has been extensively executed on the IBM BlueGene/Q located at IDRIS using up to $10^{5}$ MPI processes for grids with approximately $10^{9}$ points, see for instance 23 .

CREAMS can be run in 1D, 2D and 3D Cartesian grids and supports MPI domain decomposition in the three spatial directions. It utilises sophisticated, eighth-order accurate spatial discretisation schemes (i.e. WENO stencils) requiring four halo elements $\left(N_{h}=4\right)$. A third-order accurate time integration is performed explicitly and in three consecutive stages, which requires at least three point-to-point MPI communication per time step. In the original MPI application, halos are exchanged in one piece at the end of the integration stage. It is worth noting that each halo element consists of a (non-contiguous in memory) list of $N_{v}=5+N_{\alpha}$ double-precision real numbers, where $N_{v}$ is the total 
number of independent variables of the problem and $N_{\alpha}$ the number of species (which is about tens or even hundreds for real-world applications).

The core of CREAMS can be considered as a time-loop calling the RungeKutta 3 (rk3) subroutine that performs the time integration. This subroutine consists mainly of a loop of three iterations, inside of which there are four differentiated phases: (i) data preparation (lines 2-5), (ii) WENO stencils (line 6), (iii) data update (line 7) and (iv) halo communication (line 8) as illustrated in Code 9 corresponding to the original, MPI-only application.

Code 9: CREAMS original, MPI-only Runge-Kutta 3 subroutine.

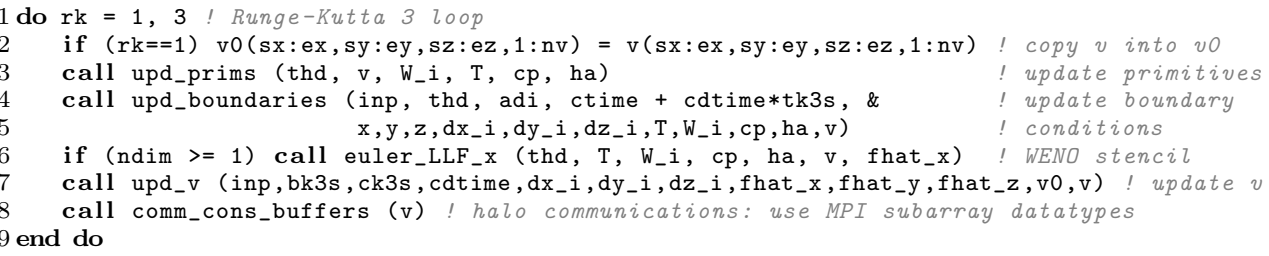

Code 9 also represents the highest level of application code, with computation and communication parts clearly differentiated, where a complete hybrid approach combining MPI domains and OmpSs-2 subdomains can be implemented. The proposed domain/subdomain methodology seeks to minimise the changes that need to be applied to the original source code, so that it results in a relatively simple refactoring. This is of great importance considering that the application contains about $10^{5}$ lines of Fortran source code. In order to define subdomains, one needs to look firstly at the MPI-only application, i.e. at the domains, in which multi-dimensional arrays representing physical variables (such as v in Code 9) are allocated in memory in the following way:

$\mathrm{v}(\mathrm{sx}-\mathrm{nh}: \mathrm{ex}+\mathrm{nh}, \mathrm{sy}-\mathrm{nh}: \mathrm{ey}+\mathrm{nh}, \mathrm{sz}-\mathrm{nh}: \mathrm{ez}+\mathrm{nh}, 1: \mathrm{nv})$

where the (defined in global memory) indexes $\mathrm{nh}$ correspond to $N_{h}$, nv to $N_{v}$ and $\mathbf{s x}$ and ex represent the starting and ending MPI domain indexes for the first spatial direction, respectively. The same applies to $y$ - and $z$ directions but, for the sake of conciseness, only the first direction is considered here. Hence a problem of size $n t x$ divided in nmpix domains has indexes $\mathbf{s x}=1$ and ex $=n t x / n m p i x$ for the first MPI domain, whilst the last one has indexes $\mathrm{sx}=1+n t x *(\mathrm{nmpix}-1) / \mathrm{nmpix}$ and ex=ntx. For example, a do-loop over half of each physical (i.e. without halos) MPI domain can be simply written as:

do $1=1$, nv; do $k=e z / 2$, ez; do $j=e y / 2$, ey; do $i=e x / 2$, ex

$\mathrm{v}(\mathrm{i}, \mathrm{j}, \mathrm{k}, \mathrm{l})=0.0$

end do; end do; end do; end do

The next step of the HDOT approach considers that each MPI domain is composed of an arbitrary number $n$ of OmpSs-2 subdomains: for a 1D problem, this is equivalent to divide the range $\mathbf{s x}-\mathrm{nh}: \mathrm{ex}+\mathrm{nh}$ into $n$ parts. Typically, the 
value of $n$ is a multiple (e.g. 2, 4, 8..) of the number of cores assigned to shared memory parallel processes. Furthermore, for 2D and 3D problems subdomain cuts must guarantee that they are always defined in contiguous portions of the original memory layout: this implies cuts in the $y$-direction for $2 \mathrm{D}$ problems and in the $z$-direction for 3D problems.

Code 10: Example of a hybrid loop in CREAMS.

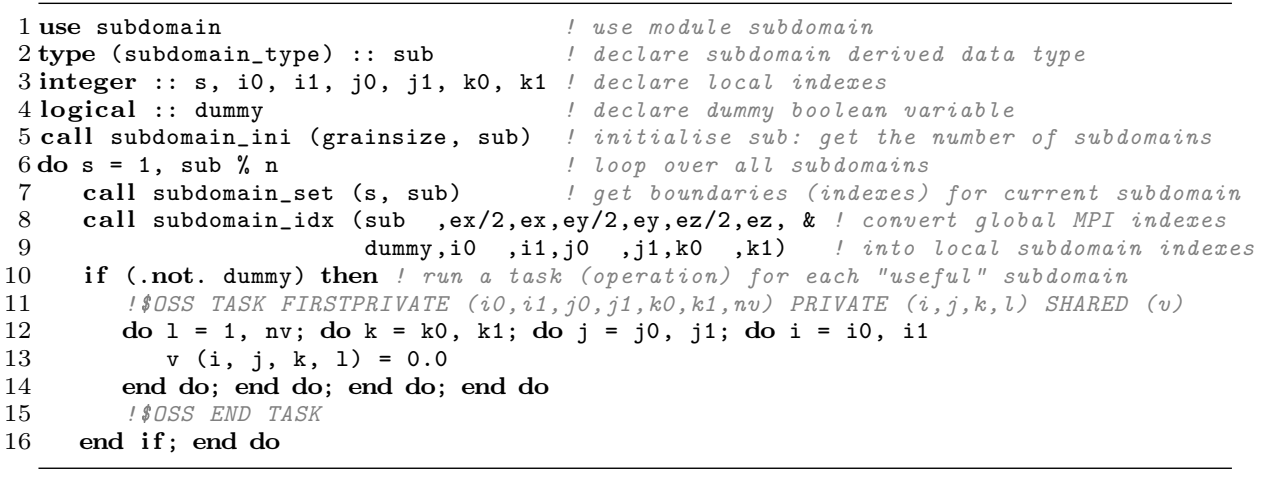

In order to effectively incorporate subdomains in the source code, it becomes necessary to define a Fortran module containing a derived data type representing the subdomain, e.g. total number of subdomains, domain ID and its boundaries (indexes), as well as functions to manipulate the data type. This approach allows writing a hybrid version of the previous nested loop as shown in Code 10. This self-explanatory code only depends on the input variable grainsize corresponding to the subdomain partition size defined by the user. Moreover, global indexes sx and ex are replaced by local indexes i0 and i1 via the function subdomain $\_i d x$. This change from global to local indexes is necessary to work with subdomains and constitutes indeed the major code refactoring.

Nevertheless, the changes to be made are pretty straightforward and only require defining local integers and passing an additional argument (sub) as a first-private copy in the corresponding subroutines, thus leaving intact the original memory layout of the application and ensuring a thread-safe execution.

Moreover, when using subdomains the new local indexes must always refer to absolute coordinates. For instance, defining a symmetry-type boundary condition at the rightmost point $\mathrm{ntx}$ using global, relative indexes:

do $1=1$, nv; do $k=s z$, ez; do $j=s y, e y ;$ do $i=1$, $n h$

$\mathrm{v}(\mathrm{ntx}+\mathrm{i}, \mathrm{j}, \mathrm{k}, \mathrm{l})=\mathrm{v}(\mathrm{ntx}-\mathrm{i}, \mathrm{j}, \mathrm{k}, \mathrm{l})$

end do; end do; end do; end do

must be replaced by local, absolute indexes:

do $1=1$, nv; do $k=k 0, k 1 ;$ do $j=j 0, j 1 ;$ do $i=i 0$, i1 ! $i 0=n t x+1$

$\mathrm{v}(\mathrm{i}, \mathrm{j}, \mathrm{k}, \mathrm{l})=\mathrm{v}(\mathrm{ntx}+\mathrm{ntx}-\mathrm{i}, \mathrm{j}, \mathrm{k}, 1)$ ! $i 1=n t x+n h$

end do; end do; end do; end do 
for subdomains indexes to be properly defined, which sometimes might be a bit counterintuitive: it is indeed much easier to perform a loop from 1 to $\mathrm{nh}$ and then count backwards and forwards from the symmetry point $\mathrm{ntx}$ as in the first example corresponding to the original, MPI-only application.

Code 11: CREAMS hybrid Runge-Kutta 3 subroutine (OmpSs-2 pragmas heavily simplified).

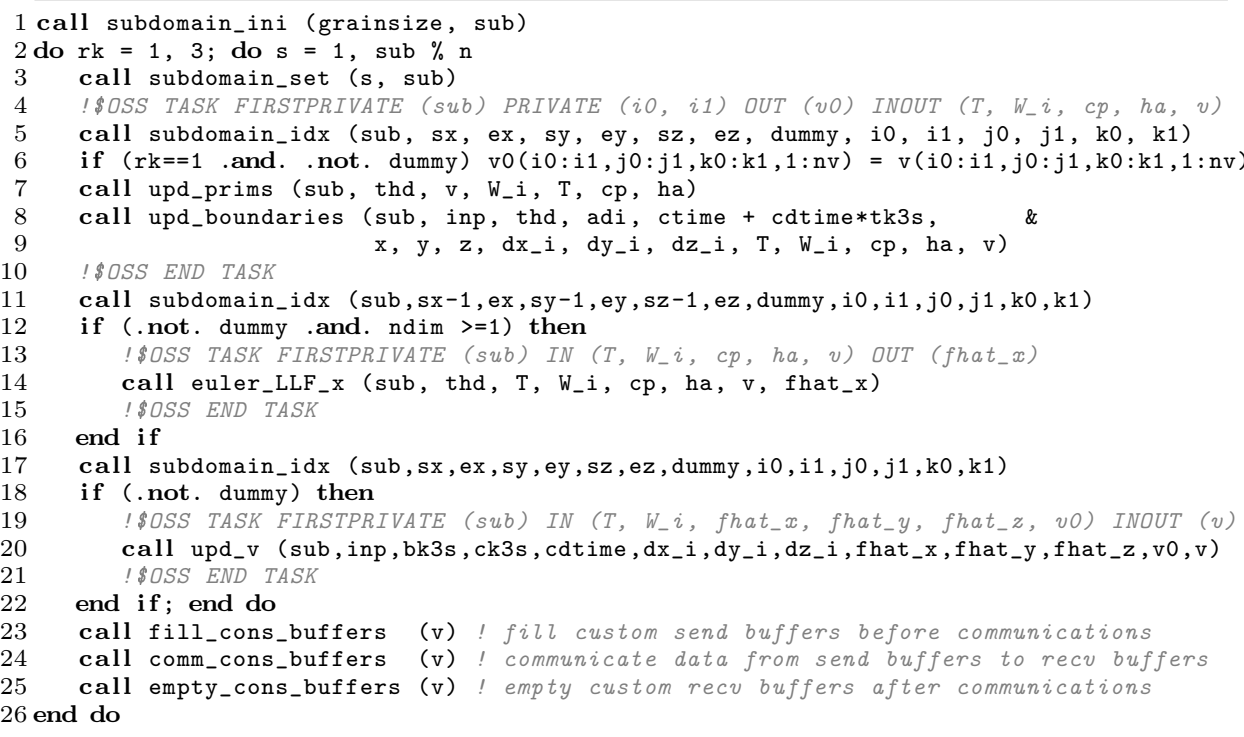

On the other hand, it is important to highlight that the creation of subdomains/tasks is not performed at low-level loops such as the one shown in Code 10, but at the highest possible level of the application code: the rk3 subroutine itself. As a result, the hybrid version of CREAMS, see Code 11. looks quite similar to the original MPI-only implementation. Leaving out pragma annotations, which are heavily simplified here due to page restriction, the application core becomes completely hybrid using only three subdomain/task groups (lines 4-10, 13-15 and 19-21 of Code 11) for computations as illustrated in Figure 5. From this figure it can be readily seen that the WENO stencils can be executed simultaneously as they are placed in the same horizontal time line. For one subdomain and 3D problems there are three simultaneous stencil executions (one in each spatial dimension), and this number doubles for two subdomains. Paraver traces depicted in Figure 6 give a better understanding of the OmpSs-2 real-time execution. The single-threaded CREAMS execution of Figure 6 reveals that WENO stencils are indeed the most computationally demanding tasks, which account for more than $95 \%$ of the total runtime on average. However, with two subdomains, there are always six busy threads computing WENO stencils in either direction and, when data needs to be prepared or updated, this number reduces to the actual number of subdomains, 
(a)

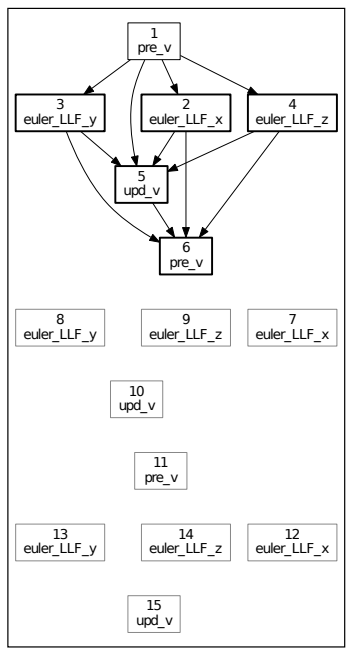

(b)

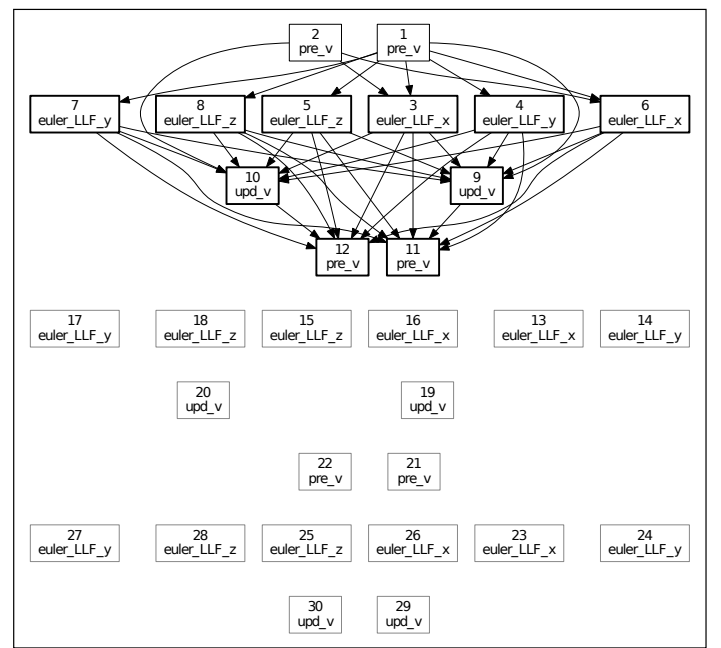

Figure 5: CREAMS dependencies graph corresponding to the first stage of the Runge-Kutta time integration (the other two are just a repetition) for (a) one OmpSs-2 subdomain and (b) two OmpSs-2 subdomains; WENO stencils (euler_LLF_*) can run in parallel from each other and pre_v and upd_v refer to data preparation and data update tasks, respectively.

following the graph patterns exposed in Figure 5.

The source code containing MPI communication also needs to be adapted to work with subdomains. In the original, MPI-only application, MPI subarray datatypes are used to duplicate halos, whilst communication (line 8 of Code 9 simply consist of calling all the non-blocking sends (MPI_ISEND) and receives (MPI_IRECV) functions with a final blocking call to MPI_WAITALL that synchronises the entire data exchange. For the hybrid version, instead of using MPI subarray datatypes, we define custom (contiguous in memory) send/recv buffers that are filled (line 23 of Code 11) and emptied (line 25 of Code 11), respectively, between an MPI communication to ensure that the data is available before sending it and also available after receiving it and used by another task. 


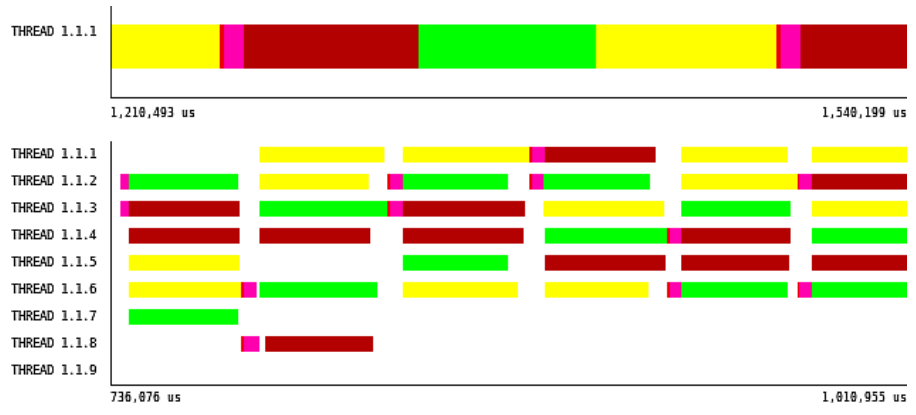

Figure 6: CREAMS Paraver trace for one OmpSs-2 subdomain forced to be executed with one thread (top) and two OmpSs-2 subdomains without thread restrictions (bottom); tasks and colours are as follows: pre_v (pink), euler_LLF_x (brown), euler_LLF_y (green), euler_LLF_z (yellow) and upd_v (red).

Code 12: CREAMS hybrid communication with East neighbours (OmpSs-2 pragmas heavily simplified).

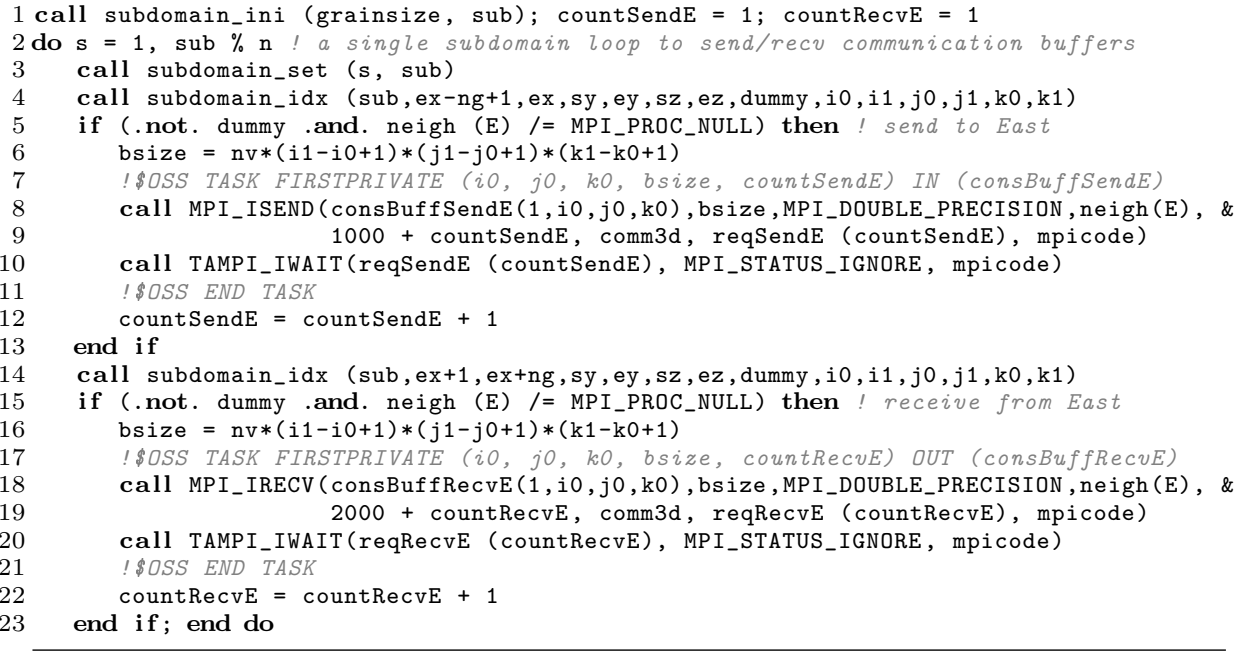

The actual MPI communication is performed by the comm_cons_buffers subroutine (line 24 of Code 11) and takes place inside subdomain tasks using the TAMPI library. As an example, the communication involving the East neighbour, i.e. the neighbour following the positive $x$-direction, is shown in Code 12. The code structure remains similar to the one used for computation tasks. Herein, the TAMPI subroutine TAMPI_IWAIT guarantees that the corresponding tasks do not get blocked waiting to receive the buffer and, instead, return immediately. This way MPI waits are completely substituted by IN and OUT pragma clauses taking place during the filling and emptying of commu- 
(a)

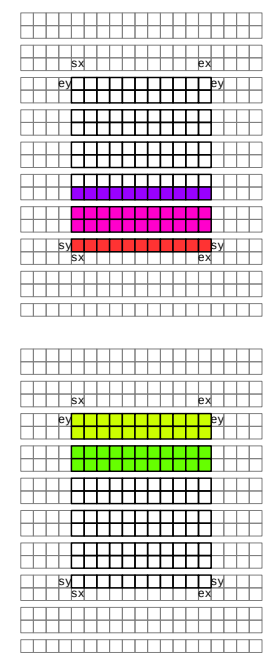

(b)

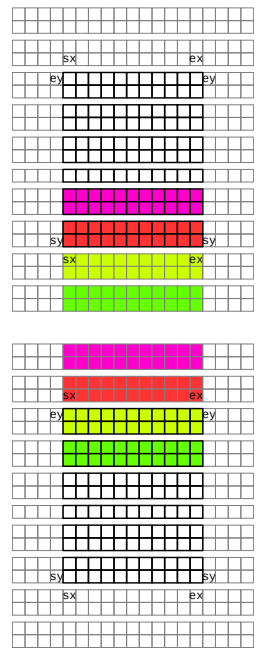

(c)

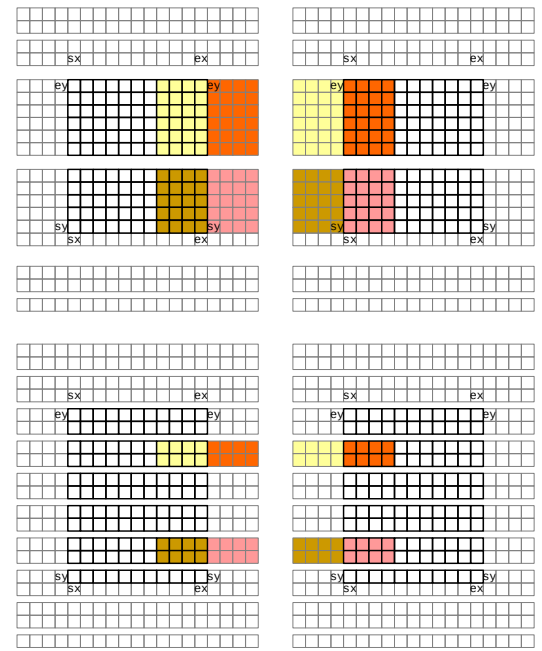

Figure 7: CREAMS possible subdomain decompositions yielding to (a) invalid parallel MPI communication, (b) valid parallel MPI communication and (c) always valid orthogonal MPI communication.

nication buffers (lines 23 and 25, respectively, of Code 11) and the generated dependencies are managed by OmpSs-2 as if they were another part of the computation. Note also that Code 11. corresponding to the hybrid version of CREAMS, will only work with a version of the MPI library with multi-threading support due to the use of TAMPI, whilst the MPI-only version is executed with a single MPI thread.

It should be noted that it is also possible to embed communication inside tasks without relying upon the TAMPI library (not shown here for the sake of conciseness). This procedure is similar to the one shown in Code 12 except that: (i) two subdomain loops are required (instead of a single one), the first loop only to send the communication buffers, and the second loop only to receive the communication buffers and where TAMPI_IWAIT is replaced by MPI_WAIT; (ii) it must be granted that all the send buffers are sent before start receiving any recv buffer. It can be readily seen that this alternative approach requires the programmer to ensure that no MPI dead locks occur by enforcing the order in which tasks/communication must be executed via blocking directives which ultimately yield an innefficient usage of MPI.

There are two important aspects regarding MPI communication with subdomains that are worth discussing. The first one deals with the communication in the direction that is parallel to subdomains, e.g. the $y$-direction in a 2D problem, see Figures $7 \mathrm{a}-\mathrm{b}$. One needs to take into account that a point-to-point send/recv communication is always asymmetric with respect to the communication edges of the two involved MPI domains. In Figure 7 a, the proposed subdomain de- 
composition is not asymmetric and hence there is not a valid correspondence between the three tasks from the top domain and the two from the bottom domain. In CREAMS the number of halo elements is $N_{h}=4$ and hence the minimum allowed grainsize values are 1, 2 (see Figure $7 \mathrm{p}$ ) or 4 to guarantee asymmetry. On the other hand, an MPI communication orthogonal to subdomain cuts does not have any constraints in terms of subdomain decomposition, see Figure 7 .

The second aspect concerns the actual implementation of MPI communication within subdomains. The proposed implementation needs three subroutines (lines 23-25 of Code 11) and therefore implies generating three groups of additional tasks and thus potentially more overhead for the OmpSs-2 runtime. A more optimised implementation could be achieved by embedding these calls inside the computation tasks, which would require modifying the pragma clauses accordingly. This implementation will be studied in a later development phase of the application as the scalability results obtained using the proposed approach already look quite promising.

Finally, Table 2 shows CREAMS scalability results obtained with the MPIonly and hybrid versions of the application. These results correspond to the Sod tube benchmark, which is commonly employed to test the robustness and accuracy of numerical schemes for compressible flows 22] and is also suitable for measuring scalability performance 24]. The dimensions of the computational domain are $N_{x} \times N_{y} \times N_{z}=20 \times 20 \times 7000$, resulting in a total of 2.8 million grid points, and runtimes values are measured after 1000 complete time steps using up to 16 nodes of the MareNostrum 4 supercomputer. Hybrid computations are set up in such a way that they only use 2 MPI domains per node (one MPI domain per socket), thus reducing the total amount of MPI messages exchanged between ranks, and each MPI domain have all the available 28 cores per socket to carry out subdomain/task computations. Even when using a single node, the hybrid version is $2.58 \%$ faster than the MPI-only version. As the number of nodes increases, this gain becomes non-negligible and, in this particular benchmark, reaches a maximum value of $13.33 \%$. This difference is mainly due to the increasing amount of communication among the 768 MPI processes (16 nodes) present in the MPI-only version compared to the only 32 MPI processes required by the hybrid version.

The above results confirm the advantages of hybrid parallel programming over the classical MPI decomposition. It is worth mentioning that, on the one hand, the communication pattern of the original MPI-only version does not allow overlapping communication with computation thus yielding suboptimal performance. On the other hand, the results of the hybrid version of CREAMS correspond to early stages of development and, consequently, better figures should be expected with the implementation of array reductions and tasks loops that are not currently fully supported in the Fortran version of OmpSs-2. Similarly, better scalability is expected with the integration of MPI communication inside computation tasks and by reducing the number of MPI processes to only one per node with an interleaved memory NUMA policy between sockets.

Finally, we would like to summarise the necessary code changes required to 
Table 2: CREAMS Sod tube parallel scalability results on MareNostrum 4; indexes M and $\mathrm{H}$ refer to the MPI-only and hybrid (MPI+OmpSs-2) CREAMS application, respectively. SI units.

\begin{tabular}{ccrrrrrr}
\hline Nodes & Runtime $_{\mathrm{M}}$ & Runtime $_{\mathrm{H}}$ & \multicolumn{1}{c}{$\mathrm{S} \cdot{ }_{\mathrm{M}}$} & \multicolumn{1}{c}{$\mathrm{S} \cdot{ }_{\mathrm{H}}$} & \multicolumn{1}{c}{ Perf. ${ }_{\mathrm{M}}$} & \multicolumn{1}{c}{ Perf. ${ }_{\mathrm{H}}$} & Gain $_{\mathrm{M} \rightarrow \mathrm{H}}$ \\
\hline 1 & 962.681 & 937.886 & 1 & 1 & $100.00 \%$ & $100.00 \%$ & $2.58 \%$ \\
2 & 485.431 & 470.240 & 1.98 & 1.99 & $99.16 \%$ & $99.72 \%$ & $3.13 \%$ \\
4 & 250.200 & 235.342 & 3.85 & 3.98 & $96.19 \%$ & $99.63 \%$ & $5.94 \%$ \\
8 & 131.880 & 118.728 & 7.30 & 7.90 & $91.25 \%$ & $98.74 \%$ & $9.97 \%$ \\
16 & 69.705 & 60.415 & 13.81 & 15.52 & $86.32 \%$ & $97.03 \%$ & $13.33 \%$ \\
\hline
\end{tabular}

apply the HDOT methodology on an MPI-only, complex application composed of hundreds of thousands of lines of source code. Firstly, to avoid rewriting the whole application from scratch, it is necessary to build a new module or class that helps handling the original data layout (where indexes are global) via subdomain/tasks (where indexes become local). This still implies some refactoring as shown in Code 10. Secondly, it is necessary to identify the highest possible level of application code to define subdomains/tasks in order to: (i) reduce the refactoring effort to the strict minimum, (ii) also reduce the total amount of tasks per execution (thus yielding better performance) and (iii) avoid leaving parts of the code serialised. By combining these two steps, subdomains/tasks will cover vast regions of the application code and basically consist of calls to other functions instead of small kernel loops as shown in Code11. Note also that OmpSs-2 pragma annotations will only be inserted at this level. Thirdly, MPI communication must also be made compatible with the specification of subdomain/tasks and hence require custom send/recv buffers if this was not originally the case (e.g. MPI subarray datatypes originally used CREAMS, which greatly simplify the use of communication buffers, cannot longer be utilised). Moreover, subdomain decomposition must be consistent with communication patterns as shown in Figure 7. Finally, there are multiple ways to perform MPI communication with tasks/subdomains, and the best implementation seeks to overlap it with computation (avoiding potential dead locks). The approach retained here achieves that with the aid of the TAMPI library, which greatly simplifies the treatment of dependencies between OmpSs-2 tasks and avoids MPI deadlocks.

\section{Conclusion and future work}

In this work, we have presented a methodology to develop hybrid applications that overcome the main issues of MPI-only and traditional hybrid MPI+OpenMP applications. The main point of this methodology is to apply the original MPI domain decomposition strategy as implemented on process-level to task-level. This way each part of the global domain assigned to an MPI rank is divided in subdomains that will be processed by OmpSs- 2 tasks. This paper describes how typical computation and communication patterns have to be modified to obtain the best performance while maximising code reuse. The hierarchical domain 
over-decomposition with tasking (HDOT) methodology also exploits synergies between MPI and OmpSs-2: on the one hand, it reuses the original MPI parallelisation strategy to expose coarse-grained parallelism inside a node; on the other hand, the OmpSs-2 data-flow execution based on fine-grained dependencies is leveraged to provide fine-grained, internode synchronisation. HDOT relies on the task-aware MPI (TAMPI) interoperability library as well as advanced support of task nesting and fine-grained dependencies provided by OmpSs-2 to minimise the structural changes required to develop a performant hybrid version. Although it would be possible to achieve similar performance improvements by (i) using the current features provided by MPI such as shared-memory, one-side communication, (ii) developing a custom user runtime on top of MPI and (iii) implementing a custom interoperatibility library. We think that this solution will be more complex, harder to use and it will require significant changes in the application structure.

HDOT has been applied to two benchmarks (Heat2D and HPCCG) and one large application (CREAMS) and the performance results have shown a clear gain in performance over other traditional approaches relying on either MPI exclusively or MPI+OpenMP. Nevertheless, additional testing with more applications will be required to further refine it. Specifically, we plan to apply this methodology to other well-known benchmarks (e.g. LULESH [25]) and to a particle-in-cell based method (similar to iPIC3D [26]) to improve our current discussion. The main goal of these future studies will be focused on finding new patterns that could be included in the current methodology, but also on looking for boundaries of this approach.

In addition, the HDOT methodology is applicable to other message passing APIs such as GASPI making use of advanced features of the OmpSs-2 runtime system. We are interested in presenting this approach as well as the discussed runtime features to the OpenMP architecture review board in order to promote their adoption for hybrid programming. We will also explore the oportunities to extend the current specification of the OpenMP detach clause in order to be more flexible with respect to the number of non-blocking services involved in the task finalisation.

\section{Acknowledgements}

This work has been developed with the support of the European Union H2020 program through the INTERTWinE project (agreement number 671602); the Severo Ochoa Program awarded by the Spanish Government (SEV-20150493); the Generalitat de Catalunya (contract 2017-SGR-1414); and the Spanish Ministry of Science and Innovation (TIN2015-65316-P, Computación de Altas Prestaciones VII). The authors gratefully acknowledge Dr. Arnaud Mura, CNRS researcher at Institut PPRIME in France, for the numerical tool CREAMS.

Finally, the manuscript has greatly benefited from the precise comments of the reviewers. 


\section{References}

[1] Message Passing Interface Forum, MPI: A message-passing interface standard. Version 3.1, University of Tennessee, 2015.

[2] T. Hoefler, J. Dinan, D. Buntinas, P. Balaji, B. Barret, R. Brightwell, W. Gropp, V. Kale, R. Thakur, Leveraging MPI's One-Sided Communication Interface for Shared-Memory Programming, in: Proceedings of the 19th European MPI Users' Group Meeting (EuroMPI 2012), ACM Press, 2012, pp. 132-141. doi:10.1007/978-3-642-33518-1_18. URL https://dl .acm.org/citation. cfm?id=2404056

[3] Y. Yan, S. Chatterjee, Z. Budimlic, V. Sarkar, Integrating MPI with Asynchronous Task Parallelism, Springer, Berlin, Heidelberg, 2011, pp. 333-336. doi:10.1007/978-3-642-24449-0_41. URL http://link. springer . com/10.1007/978-3-642-24449-0\{_\}41

[4] R. F. Barrett, D. T. Stark, C. T. Vaughan, R. E. Grant, S. L. Olivier, K. T. Pedretti, Toward an evolutionary task parallel integrated MPI $+\mathrm{X}$ programming model, in: Proceedings of the Sixth International Workshop on Programming Models and Applications for Multicores and Manycores PMAM '15, 2015, pp. 30-39. doi:10.1145/2712386.2712388.

[5] S. Chatterjee, S. Tasirlar, Z. Budimlic, V. Cave, M. Chabbi, M. Grossman, V. Sarkar, Y. Yan, Integrating Asynchronous Task Parallelism with MPI, in: 2013 IEEE 27th International Symposium on Parallel and Distributed Processing, IEEE, 2013, pp. 712-725. doi:10.1109/IPDPS.2013.78. URL http://ieeexplore.ieee.org/document/6569856/

[6] OpenMP Architecture Review Board, OpenMP Application Programming Interface, version 4.5 (2015).

[7] OpenMP Architecture Review Board, OpenMP Application Programming Interface, version 3.0 (2008).

[8] Barcelona Supercomputing Center, OmpSs-2 Specification (Accessed on: April, 8th 2019). URL https://pm.bsc.es/ftp/ompss-2/doc/spec/

[9] K. Sala, J. Bellon, P. Farre, X. Teruel, J. M. Perez, A. J. Pena, D. Holmes, V. Beltran, J. Labarta, Improving the Interoperability between MPI and Task-Based Programming Models, in: Proceedings of the 25th European MPI Users' Group Meeting on - EuroMPI 2018, ACM Press, Barcelona, Spain, 2018.

[10] K. Sala, X. Teruel, J. M. Perez, A. J. Peña, V. Beltran, J. Labarta, Integrating blocking and non-blocking mpi primitives with task-based programming models, Parallel Computing (2018). doi:https://doi.org/10.1016/j.parco.2018.12.008. 
URL S0167819118303326

[11] V. Marjanović, J. Labarta, E. Ayguadé, M. Valero, Overlapping communication and computation by using a hybrid MPI/SMPSs approach, in: Proceedings of the 24th ACM International Conference on Supercomputing, 2010, pp. 5-16. doi:10.1145/1810085.1810091.

[12] D. Doerfler, R. Brightwell, Measuring MPI send and receive overhead and application availability in high performance network interfaces, in: Recent Advances in Parallel Virtual Machine and Message Passing Interface, Springer, Berlin, Heidelberg, 2006, pp. 331-338. doi:10.1007/11846802_ 46.

URL http://link.springer.com/chapter/10.1007/11846802\{_\}46

[13] T. Hoefler, A. Lumsdaine, W. Rehm, Implementation and performance analysis of non-blocking collective operations for MPI, in: Proceedings of the 2007 ACM/IEEE Conference on Supercomputing - SC '07, ACM Press, New York, New York, USA, 2007, p. 1. doi:10.1145/1362622.1362692. URL http://portal . acm. org/citation . cfm?doid=1362622.1362692

[14] R. L. Graham, S. Poole, P. Shamis, G. Bloch, N. Bloch, H. Chapman, M. Kagan, A. Shahar, I. Rabinovitz, G. Shainer, Overlapping computation and communication: Barrier algorithms and ConnectX-2 CORE-Direct capabilities, in: 2010 IEEE International Symposium on Parallel \& Distributed Processing, Workshops and Phd Forum (IPDPSW), IEEE, 2010, pp. 1-8. doi:10.1109/IPDPSW.2010.5470854.

URL http://ieeexplore. ieee.org/document/5470854/

[15] C.-Q. Yang, B. Miller, Critical path analysis for the execution of parallel and distributed programs, in: Proceedings. The 8th International Conference on Distributed, IEEE Comput. Soc. Press, 1988, pp. 366-373. doi:10.1109/DCS.1988.12538.

URL http://ieeexplore.ieee.org/document/12538/

[16] J. Hollingsworth, Critical path profiling of message passing and sharedmemory programs, IEEE Transactions on Parallel and Distributed Systems 9 (10) (1998) 1029-1040. doi:10.1109/71.730530. URL http://ieeexplore.ieee.org/document/730530/

[17] M. Schulz, Extracting Critical Path Graphs from MPI Applications, in: 2005 IEEE International Conference on Cluster Computing, IEEE, 2005, pp. 1-10. doi:10.1109/CLUSTR.2005.347035.

URL http://ieeexplore .ieee.org/document/4154078/

[18] F. Schmitt, R. Dietrich, G. Juckeland, Scalable Critical Path Analysis for Hybrid MPI-CUDA Applications, in: 2014 IEEE International Parallel \& Distributed Processing Symposium Workshops, IEEE, 2014, pp. 908-915. 
doi:10.1109/IPDPSW. 2014.103.

URL http://ieeexplore .ieee.org/document/6969479/

[19] R. Thakur, P. Balaji, D. Buntinas, D. Goodell, T. Hoefler, S. Kumar, E. Lusk, J. L. Träff, Mpi at exascale.

[20] J. M. Perez, V. Beltran, J. Labarta, E. Ayguadé, Improving the integration of task nesting and dependencies in openmp, in: 2017 IEEE International Parallel and Distributed Processing Symposium (IPDPS), 2017, pp. 809818.

[21] M. A. Heroux, D. W. Doerfler, P. S. Crozier, J. M. Willenbring, H. C. Edwards, A. Williams, M. Rajan, E. R. Keiter, H. K. Thornquist, R. W. Numrich, Improving Performance via Mini-applications, Tech. Rep. SAND20095574, Sandia National Laboratories (2009).

[22] P. J. M. Ferrer, R. Buttay, G. Lehnasch, A. Mura, A detailed verification procedure for compressible reactive multicomponent Navier-Stokes solvers, Computers \& Fluids 89 (2014) 88-110. doi:10.1016/j.compfluid.2013. 10.014

URL https : //doi.org/10.1016/j.compfluid.2013.10.014

[23] P. J. M. Ferrer, G. Lehnasch, A. Mura, Compressibility and heat release effects in high-speed reactive mixing layers II. Structure of the stabilization zone and modeling issues relevant to turbulent combustion in supersonic flows, Combustion and Flame 180 (2017) 304-320. doi: 10.1016/j.combustflame.2016.09.009.

URL https://doi.org/10.1016/j.combustflame.2016.09.009

[24] S. Macià, S. Mateo, P. J. Martínez-Ferrer, V. Beltran, D. Mira, E. Ayguadé, Saiph: Towards a DSL for high-performance computational fluid dynamics, in: Proceedings of the Real World Domain Specific Languages Workshop 2018, RWDSL2018, ACM, New York, NY, USA, 2018, pp. 6:1-6:10. doi: 10.1145/3183895.3183896.

URL http://doi.acm.org/10.1145/3183895.3183896

[25] Hydrodynamics Challenge Problem, Lawrence Livermore National Laboratory, Tech. Rep. LLNL-TR-490254.

[26] S. Markidis, G. Lapenta, Rizwan-uddin, Multi-scale simulations of plasma with ipic3d, Mathematics and Computers in Simulation 80 (7) (2010) 1509 - 1519, multiscale modeling of moving interfaces in materials. doi:https://doi.org/10.1016/j.matcom.2009.08.038.

URL http://www.sciencedirect.com/science/article/pii/ S0378475409002444 